Errata of the article:

\title{
Eigenfunctions of Two-Scale Difference Equations with Dilation Parameter and Infinite Products
}

\author{
by \\ Manfred Krüppel \\ in RM, vol. 47 (2005) pp. 93-114
}

As editor I again have to apologize that due to incompatibility of different TEX programs some equality signs are eliminated by our programs. Below you will find the corrections.

Heinrich Wefelscheid

page 93 , formula (1.1)

$$
\lambda \psi\left(\frac{t}{\alpha}\right)=\sum_{\nu=0}^{\infty} d_{\nu} \psi(t-\nu) \quad(t \in \mathbb{R})
$$

page 95 , formula $(2.2)$

$$
\frac{E_{\nu}(w)}{(1-w)^{\nu+1}}=\sum_{\mu=1}^{\infty} \mu^{\nu} w^{\mu-1} \quad\left(\nu \in \mathbb{N}_{0}\right)
$$

page 96 , formula after (2.4)

$$
\sum_{\mu=1}^{\infty} q_{n}(a k+a \mu) w^{k+\mu-1}=w^{k} \sum_{\nu=0}^{n} \frac{a^{\nu} q_{n}^{(\nu)}(a k)}{\nu !} \frac{E_{\nu}(w)}{(1-w)^{\nu+1}} \quad(|w|<1)
$$

page 96 , formula after (2.5)

$$
\lambda \psi_{n}\left(\frac{\mu}{\alpha}\right)=\sum_{\nu=0}^{\mu-1} d_{\nu} \psi_{n}(\mu-\nu)
$$

page 97 , formula (2.9)

$$
\lambda \sum_{\nu=0}^{M_{0}} \beta_{\nu} \psi\left(\frac{t-\nu}{\alpha}\right)=\sum_{\nu=0}^{N_{0}} \alpha_{\nu} \psi(t-\nu) \quad(t \in \mathbb{R})
$$


page 97 , formula $(3.1)$

$$
\mathcal{L}\left\{\psi_{-1}\right\}=\prod_{\nu=1}^{\infty} T\left(e^{-z / \alpha^{\nu}}\right)
$$

page 97 , formula after $(3.2)$

$$
\mathcal{L}\left\{\psi^{(-1)}\right\}=\frac{1}{z} \mathcal{L}\{\psi\}=\frac{1}{z} \Psi(z)=\frac{c}{z}+\frac{1}{z}(\Psi(z)-c) \quad(\operatorname{Re} z>0)
$$

page 99 , formula before (4.1)

$$
G(\alpha z)=\prod_{\nu=0}^{\infty} \frac{P_{0}\left(e^{-z / \alpha^{\nu}}\right)}{Q_{0}\left(e^{-z / \alpha^{\nu}}\right)}
$$

page 100 , formula (4.5)

$$
G\left(2^{k+1} z\right)=G(z) \prod_{j=0}^{k} \frac{P_{0}\left(e^{-2^{j} z}\right)}{Q_{0}\left(e^{-2^{j} z}\right)}=G(z) \frac{P_{0}\left(e^{-2^{k} z}\right) \ldots P_{k-1}\left(e^{-2 z}\right) P_{k}\left(e^{-z}\right)}{Q_{0}\left(e^{-z}\right) \ldots Q_{k-1}\left(e^{-z}\right) Q_{k}\left(e^{-z}\right)}
$$

page 103 , formula $(5.9)$

$$
w^{q}-\zeta_{k}=\prod_{\ell=0}^{q-1}\left(w-\eta_{k} e^{2 \pi i \ell / q}\right)
$$

page 103 , formula after (5.9)

$$
\prod_{k=0}^{p-1}\left(w^{q}-\zeta_{k}\right)=\prod_{k=0}^{p-1} \prod_{\ell=0}^{q-1}\left(w-\xi_{0} e^{2 \pi i k / p} e^{2 \pi i \ell / q}\right)=w^{p q}-\xi_{0}^{p q}
$$

page 104 , formula after $(5.10)$

$$
G(z)=\prod_{\mu=1}^{m} Q\left(e^{-p z / \alpha^{\mu}}\right) \prod_{\nu=1}^{\infty} P\left(e^{-z / \alpha^{\nu}}\right)
$$

page 104 , formula (5.11)

$$
G(\alpha z)=Y_{0}(z) \prod_{\nu=0}^{\infty} \frac{P\left(e^{-z / \alpha^{\nu}}\right)}{R\left(e^{-z / \alpha^{\nu}}\right)}
$$

page 105 , last formula

$$
w^{p^{\ell}}-\zeta=(1-\zeta) f_{1}\left(w^{p^{\ell-1}}\right) f_{2}\left(w^{p^{\ell-2}}\right) \ldots f_{\ell}(w)
$$

page 107 , formula $(6.6)$

$$
\sigma_{L}=q \sum_{\mu=0}^{m-1} M \alpha^{\mu}=M \frac{p-q}{\alpha-1}
$$

page 108 , formula $(7.2)$

$$
\lambda \varphi\left(\frac{t}{\alpha}\right)=\sum_{\nu=0}^{N} c_{\nu} \varphi(t-\nu) \quad(t \in \mathbb{R})
$$


page 109 , formula before (7.7)

$$
\Psi(\alpha z)=S\left(e^{-\alpha z}\right) \Phi(\alpha z)=\frac{Q\left(e^{-p z}\right)}{Q\left(e^{-q z}\right)} S\left(e^{-z}\right) P\left(e^{-z}\right) \Phi(z)
$$

page 111 , formula after (8.6)

$$
\sum_{n=0}^{\infty} \gamma_{n} z^{n}=\sum_{\ell=0}^{L} s_{\ell} e^{-\sigma_{\ell} z} \sum_{\nu=0}^{\infty} a_{\nu} z^{\nu}
$$

